\title{
Bacterial adherence to mucosal epithelium in the upper airways has less significance than believed Anders Ebenfelt*
}

\author{
Address: Department of Otorhinolaryngology, Head \& Neck Surgery, Sahlgrenska University Hospital, S-413 45 Göteborg, Sweden \\ Email: Anders Ebenfelt* - anders.ebenfelt@mailer.gu.se \\ * Corresponding author
}

Published: 9 June 2003

Journal of Negative Results in BioMedicine 2003, 2:3

This article is available from: http://www.jnrbm.com/content/2/I/3

(c) 2003 Ebenfelt; licensee BioMed Central Ltd. This is an Open Access article: verbatim copying and redistribution of this article are permitted in all media for any purpose, provided this notice is preserved along with the article's original URL.
Received: 4 October 2002

Accepted: 9 June 2003

\begin{abstract}
Background: Bacterial adherence to the upper airway epithelium is considered to be an important phenomenon in the pathogenesis of infections. However, the evidence for the hypothesis that bacterial adherence to mucosal epithelial cells has significance for pathogenesis of mucosal infections is based on studies using indirect techniques. We could find no biopsy studies with direct ocular observations of significant numbers of bacteria adhering to upper airway mucosal epithelial cells either in health or during disease.

Results: We studied specimens from healthy and infected tonsillar epithelium and specimens from the soft palate epithelium obtained by surgery. The specimens were examined by TEM. In the vast majority of specimens, we found no bacteria adhering to the epithelial cells in the mucosal line regardless of whether the patient was infected or not. Bacteria adhering to shed epithelial cells were seen in higher numbers. Furthermore, as bacteria are small compared to epithelial cells, we calculated the risk of overlooking every adhered bacteria in a section if bacterial adherence was such a significant phenomenon as earlier suggested. We found this risk to be very small.

Conclusion: We conclude that bacterial adherence to mucosal surface epithelial cells is not a significant phenomenon, either in healthy mucosa in the upper airways or during infection. This is also in line with our earlier results, where we have shown that the site for the infectious process in pharyngotonsillitis is in the secretion on the tonsillar mucosal surface.
\end{abstract}

\section{Background}

Bacterial adherence to the epithelium in the upper airways has long been considered to be an important phenomenon. It is agreed that bacteria, to be infectious, have to first adhere to the mucosal epithelium and then invade the tissue [1-8]. Also, bacterial adherence is considered to be a normal physiological phenomenon in healthy mucosa $[4,5,9-11]$. The number of adhered bacteria differs between different locations in the mucosa of the mouth and pharynx but Gibbons reports that there are on average five to twenty adhered bacteria per epithelial cell on the human mucosa in the cheek and palate in health [9]. Concerning disease, Stenfors reports that about $50 \%$ of the tonsillar epithelial cells have more than ten adhering bacteria during acute pharyngotonsillitis [5].

However, the evidence that bacterial adherence to the epithelial cells on the mucosal surface is an important phenomenon, either in the pathogenesis of infections or in health, is not very strong. In almost all other studies concerning bacterial adherence to mucosal epithelium, including those referred to above, epithelial cells have 
been obtained by the scrape or brush technique and then examined by direct observation of adhering bacteria or used in an in vitro experiment $[3,5,9,12-14]$. One further study was performed on tissue using an in vitro model [2]. We could find no biopsy study where direct ocular observation of bacteria adhering to the mucosal surface is described to a significant extent. We recently studied tonsillar epithelium by TEM and could not confirm that bacterial adherence was a frequent phenomenon either in healthy tonsils or in infected tonsils [15]. We also found that during acute pharyngotonsillitis, the site for the infectious process, defined as the place where bacteria are attacked and phagocytized by neutrophils, was located in the secretion outside the mucosal surface. Thus, bacterial adherence to the mucosal surface should not be decisive for infection.

The present study was performed to further evaluate the significance of bacterial adherence in health and during disease. By transmission electron microscopy (TEM), we examined the mucosal surfaces of infected tonsils, non infected tonsils and soft palates. We also calculated the possibility of finding the adhered bacteria in these sections if the number of bacteria adhering to the epithelium was really as huge as proposed by other authors $[5,9]$.

\section{Methods}

\section{Patients}

Six patients with acute pharyngotonsillitis subjected to acute tonsilectomy due to peritonsillar abscess took part in the study. All patients showed clinically manifest acute pharyngotonsillitis as signified by a red, swollen mucosa, sore throat and elevated body temperature. Before surgery the patients had received antibiotics for 0-48 hours, table 1.

Ten patients were tonsilectomized due to recurrent pharyngotonsillitis. They did not have any tonsillar infection and were not treated with antibiotics during three months before surgery.

Ten patients were tonsilectomized due to snoring problems. These patients had not experienced any infections of the tonsils for the last five years and had not been treated with antibiotics during three months before surgery.

Another five patients underwent uvulopalatoplasty due to snoring problems. The uvulas were used for the study. None of the patients had received antibiotics during three months before surgery.

The tonsillectomized patients are also included in an earlier work, except for one further patient with acute pharyngotonsillitis [15].
Table I: Preoperative antibiotic treatment for the patients suffering from acute pharyngotonsillitis with quinsy

\begin{tabular}{llc}
\hline Patient & Antibiotics used & $\begin{array}{c}\text { Duration before } \\
\text { treatment }\end{array}$ \\
\hline A & Benzylpenicillin i.v. & I hour \\
B & Benzylpenicillin i.v. & 4 hours \\
C & Benzylpenicillin i.v. & 17 hours \\
D & Benzylpenicillin i.v. & 24 hours \\
E & Cefuroxime i.v. + metronidazole i.v. & 24 hours \\
F & Benzylpenicillin i.v. & 48 hours
\end{tabular}

\section{Sampling technique}

Immediately after excision, the tonsils were gently cut in approximately $3 \mathrm{~mm}$ thick slices. One slice from each tonsil was immerse in glutaraldehyde for further processing for transmission electron microscopy. The excised uvulas were directly immersed in glutaraldehyde for further processing for transmission electron microscopy.

\section{Tissue preparation}

The tissue samples were oriented and cut in such a manner as to allow observation of the mucosal surfaces. The tissue was fixed in $2.5 \%$ glutaraldehyde in $0.05 \mathrm{M}$ sodium cacodylate buffer for 3 days and then postfixed in $1 \%$ osmium tetroxide in cacodylate buffer for 1 hour followed by dehydration in a graded series of ethanol and propylene oxide. The samples were embedded in an epoxy resin. One micrometer thick sections were used for selection of appropriate areas, which in the tonsils also included a crypt. These areas were used for ultrathin sectioning (about $60 \mathrm{~nm}$ thickness).

The ultrathin sections were examined in a Philips EM 400 transmission electron microscope. At least six sections from each specimen were examined. Only sections with a mucosal line consisting of at least 50 epithelial cells were regarded as conclusive. The number and location of bacteria and the extent of bacterial adherence to epithelial cells were noted and then documented photographically.

\section{Calculation of the probability of overlooking adhered bacteria}

The propability of not observing any adhered bacteria on the epithelial surface in one section if bacterial adherence were as frequent as earlier described $[5,9]$ was calculated.

\section{Results \\ Acutely infected tonsils}

From each of the patients with acute infections, we obtained 6 sections which fulfilled the criteria to be conclusive. Only in one of these patients was bacterial adherence to epithelial cells in the mucosal surface seen and in 


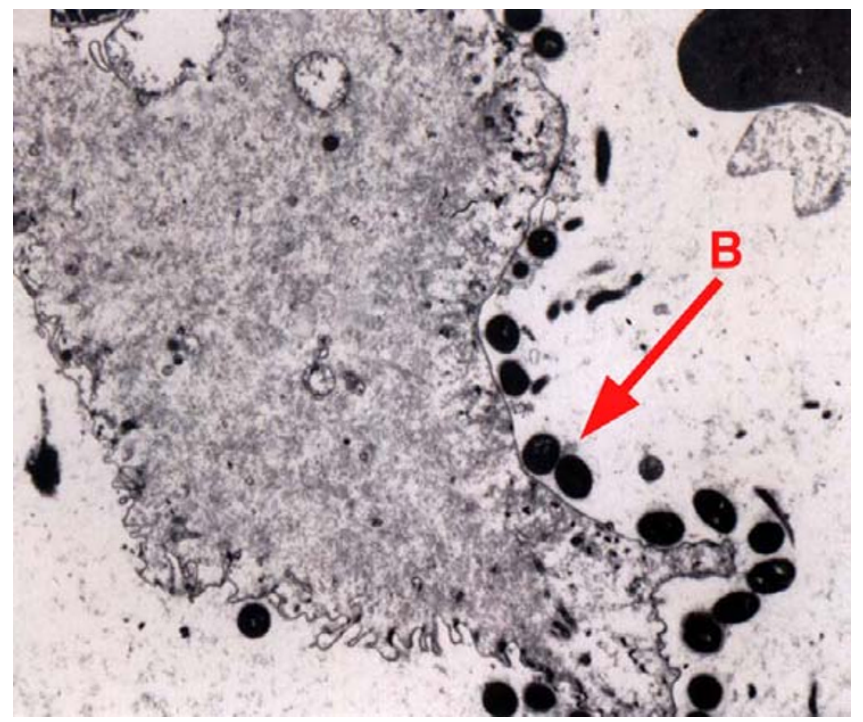

Figure I

Specimen from a patient with acute tonsillitis. A shed epithelial cell is seen. Several bacteria (labelled B) are adhering to it.

that case only one bacteria in one section. Bacterial adherence to shed epithelial cells was seen in specimens from several patients and in these specimens numerous bacteria were adhered, figure 1 . Bacteria were also present in the secretion outside the mucosal surface, figure 2.

\section{Recurrently infected tonsils}

From each of the patients with recurrent pharyngotonsilltis infections, we obtained 6 sections which fulfilled the criteria to be conclusive. Bacterial adherence to tonsillar epithelium in the mucosal surface was not observed. Bacterial adherence to shed epithelial cells was seen in specimens from some patients. Bacteria were also present in the secretion outside the mucosal surface.

\section{Healthy tonsils}

From each of the patients tonsilectomized due to snoring, we obtained 6 sections which fulfilled the criteria to be conclusive. Bacterial adherence to epithelial cells on the mucosal surface was not seen. Bacteria were seen adhered to shed epithelial cells in some cases. Also in these sections, bacteria were present in the secretion outside the mucosal surface.

\section{Soft palate}

From each of the uvuloectomized patients, we obtained at least 3 sections which fulfilled the criteria to be conclusive, with a total of 19 sections. Bacterial adherence was only observed in one of these sections and in that case two bacteria adhered to two different epithelial cells.

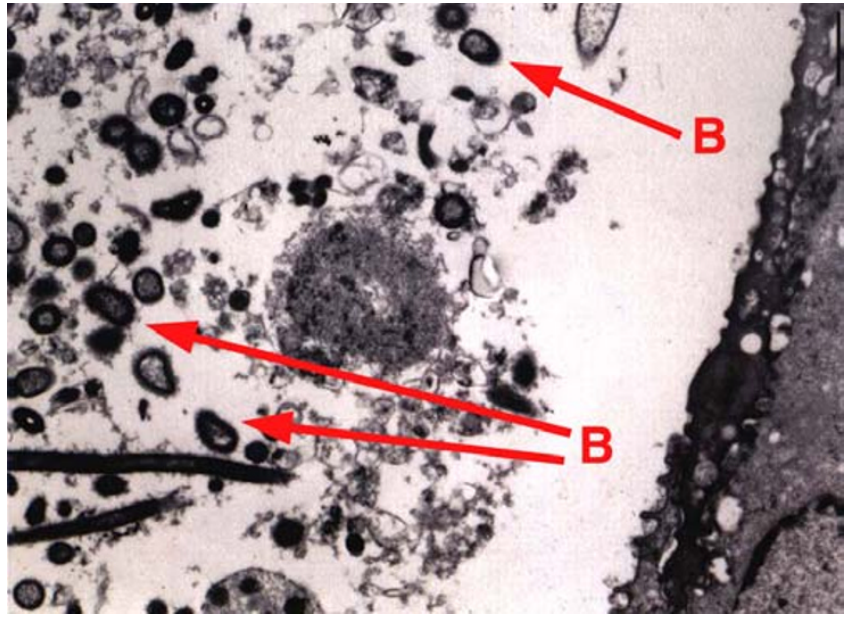

Figure 2

Specimen from a patient with acute pharyngotonsillitis. Numerous bacteria (labelled B) are located in the crypt lumen, which also contains cell debris. No bacteria are adhering to the mucosal epithelium.

\section{Calculation of the probability of overlooking adhering bacteria}

According to Stenfors, about $50 \%$ of the epithelial cells should have 11 bacteria or more adhering to them in a patient with acute pharyngotonsillitis [5]. We set the thickness of the bacteria to one micrometer and the diameter of the epithelial cell to eighty micrometers. That means that the probability of not observing a bacteria present in an indefinite thin section of an epithelial cell is 0.9875 and slightly less in a substantially thicker section. Thus, the probability of missing all (eleven) adhering bacteria on one cell is $0.9875^{11}=0.87$. In a section with 50 epithelial cells, where 25 have 11 bacteria adhering to them, the probablity of overlooking all bacteria in that single section is less than $0.87^{25}=0.03$.

According to Gibbons [9], at least five bacteria per epithelial cell are adhered to the epithelium in health. The probability of missing one adhered bacteria in an indefinite thin section of a cell is again 0.9875 . The probability of missing 250 bacteria in a section consisting of 50 cells is thus $0.9875^{250}=0.04$.

\section{Discussion}

By TEM, we have examined 36 sections from the tonsillar mucosal surface of patients with acute pharyngotonsilltis and 120 sections from patients without ongoing acute pharyngotonsilltis. We have also examined 19 sections from the mucosal surface of the soft palate. Only in two sections (one tonsillar and one from the soft palate) could 
we demonstrate bacteria adhering to the epithelial cells on the mucosal surfaces. In the vast majority of the sections, we could not observe any bacteria adhering to the epithelial surface.

As bacteria are small, we have calculated the probability that the method used was the reason why we did not find them. The calculation shows, however, that the probablity of missing every adhering bacteria in a TEM section is low if the earlier estimated level of bacterial adherence in health and during disease $[5,9]$ is correct. We can thereby conclude that the small size of bacteria is not the main reason for the lack of observations of bacterial adherence.

Whereas we only found a few bacteria adhering to epithelial cells in the mucosal line, bacterial adherence to shed epithelial cells was a far more common phenomenon. This indicates that the technique used seems to work insofar as the fixation procedure does not draw all bacteria away from the epithelial cells. If the fixation technique is the reason that bacteria are not present on the surfaces of intact epithelium, the fixation procedure should also have drawn the bacteria away from the shed epithelial cells. Also, the adherence on the shed cells shows that the phenomenon of bacterial adherence is visible by TEM. The fixation procedure used is the normal one for examining mucosal surfaces and is considered to be adequate for that purpose. It would have been even better to have some kind of positive control, that is samples from a living mucosal surface where bacteria are known to adhere. Unfortunately, we could not find such a mucosal surface and it is questionable if it exists.

Thus, as the number of sections is large, the probability of overlooking all adhering bacteria if existing as a significant phenomenon is low, and as the method can discriminate bacterial adherence, the result strongly indicates that bacterial adherence to mucosal surfaces in health and during disease is an overestimated phenomenon.

Bacterial adherence to epithelial cells is described in numerous studies $[2-6,9,10,12-14]$. However, only in two studies is direct observation of bacteria adhering to the epithelial surface in a biopsy reported $[6,16]$. In those studies, the numbers of bacteria adhering to the epithelium are not described. In all other studies concerning bacterial adherence to mucosal epithelium, epithelial cells were obtained by the scrape or brush technique and then examined by direct observation of adhering bacteria or used in an in vitro experiment $[3,5,9,12-14]$. One further study was performed on tissue using an in vitro model [2]. These studies cannot be considered to prove the existence of bacterial adherence to intact mucosal epithelium in vivo. The scrape and brush techniques used are usually performed with a small brush, gently brushing the epithe- lium without damaging the surface. If the surface is damaged in order to get huge numbers of epithelial cells, one would in these cases also get huge number of red blood corpuscles as small vessels are very near the surface. This is not the case in the cited studies as blood should have disturbed the outcome. That means that the epithelial cells collected by brush and scrape techniques would probably mainly be shed cells from the secretion and to a smaller extent epithelial cells from the epithelial layer. This is further supported by our results earlier reported about the cellular content in secretion, performed with a very smooth imprint technique, showing huge numbers of epithelial cells in the secretion during health and disease [15]. So, scrape and brush techniques do yield a huge number of shed epithelial cells and an unknown, probably low, number of epithelial surface cells and are not proof of bacterial adherence to the epithelial surface. Again, in the present study we found bacterial adherence to shed epithelial cells and, with this in mind, we suspect that the bacterial adherence reported in other studies also describes bacterial adherence to shed epithelial cells.

Several studies have been performed concerning structures on the epithelial cells which are considered to be receptors for bacterial adherence. However, some of these receptors are also present in the secretion $[9,10]$, which is the location where we observed the bacteria. We therefore assume that the bacteria have the ability to adhere to the secretion as well as to the epithelial cells. When studies are done by means of in vitro experiments, there is no secretion present, which is the probable explanation for the difference between our results and those in the abovecited studies showing bacterial adherence to epithelial cells in vitro. The preference for the bacteria to adhere to the secretion rather than the epithelial line seen in the present study could then be explained by defense mechanisms of the epithelial cells or just by bigger numbers of receptors in the secretion.

As bacterial adherence to the mucosal surface is generally considered to be the first step in infection, followed by invasion of the parenchyma $[1,4,7,8]$, our results mean that one has to reconsider the pathogenesis of mucosal infections. We have shown in earlier studies regarding pharyngotonsillitis that the site for the infectious process, defined as the site where neutrophils attack and destroy bacteria, thereby causing inflammation, is in the secretion on the mucosal surface $[15,17-19]$. In those studies, we did not observe any bacteria in the parenchyma. Thus, bacterial adherence to the mucosal surface is not necessary for the pathogenesis of infections. However, bacterial adherence may still be an important step in infection, but if so, one should direct one's attention to the bacterial adherence to the structures and cells in the secretion. 


\section{Conclusion}

Bacterial adherence to mucosal epithelial cells seems to be an overestimated phenomenon in the pathogenesis of infection and also in health. The pathogenesis of infections in the upper airways should instead be studied with the focus on the secretion on the mucosal surfaces.

\section{Acknowledgements}

We are grateful to the Swedish Medical Research Council and Göteborg Medical Society for supporting this study.

\section{References}

I. Abraham SN and Beachey $\mathrm{EH}$ : Host defences against adhesion of bacteria to mucosal surfaces In: Advances in Host Defense Mechanisms Volume 4. Edited by: Gallin Jl, Fauci AS. New York: Raven; 19856387.

2. Dudley JP and Cherry JD: Demonstration of the adherence of Streptococcus pyogenes to the surface of human tonsillar tissue Am-J-Otolaryngol I980, I (4):269-74.

3. Galioto GB, Mevio E and Maserati R: Bacterial adherence and upper respiratory tract disease: a correlation between $S$. pyogenes attachment and recurrent throat infections Acta Otolaryngol Suppl Stockh 1988, 454: 167-74.

4. Beachey $\mathrm{EH}$ and Courtney HS: Bacterial adherence of group $\mathbf{A}$ streptococci to mucosal surfaces Respiration 1989, 55(suppl I):33-40.

5. Stenfors L-E and Räisänen S: Attachment of bacteria to tonsillar epithelium during acute tonsillit The journal of Laryngology and Otology 1991, 105:29-32.

6. Fredriksen F, Räisänen S, Myklebust R and Stenfors L: Bacterial adherence to the surface and isolated cell epithelium of the palatine tonsils Acta Otolaryngol (Stockh) 1996, I I 6:620-626.

7. Gibbons RJ: Adherence of bacteria to host tissue In: Microbiology Edited by: Schlessinger D. Washington DC: American Society for Microbiology; 1977:395-406.

8. Hansson LÀ, Andersson B and Carlsson B: Defence of mucous membranes by antibodies, receptor analouges and non-specific host factors Infection 1985, I3(Suppl 2):SI66-170.

9. Gibbons RJ and van Houte J: Bacterial adherence in oral microbial ecology Am Rev Microbiol 1975, 29:19-44.

10. Niederman MS: Bacterial adherence as a mechanism of airway colonization Eur J Clin Microbiol Infect Dis 1989, 8(I): I5-20.

II. Reynolds HY: Bacterial adherence to respiratory tract mucosa-A dynamic interaction leading to colonization Seminars in Resp Inf 1987, 2(1):8-19.

12. Fainstein $\vee$ and Musher DM: Bacterial adherence to pharyngeal cells in smokers, nonsmokers, and chronic bronchitis Infect Immunity 1979, 26(I): I78-182.

13. Stenfors L-E and Räisänen S: Is attachement of bacteria to the epithelial cells of the nasopharynx the key to otitis media? Int J Ped Otorhinolaryngol 1991, 22:1-8.

14. Stenfors LE, Raisanen S and Rantala I: In vivo attachment of group A streptococci to tonsillar epithelium during acute tonsillitis Scand J Infect Dis 1991, 23(3):309-I3.

15. Ebenfelt A, Ericson LE and Lundberg C: Acute pharyngotonsillitis is an infection restricted to the crypt and surface secretion Acta Otolaryngol (Stockh) 1998, I I 8:264-27I.

16. Hokonohara M, Yoshinaga M, Inoue $\mathrm{H}$, Haraguchi $\mathrm{T}$ and Miyata $\mathrm{K}$ : Experimental studies on the initial focus of invasion of group A streptococci Acta Otolaryngol 1988, Suppl 454:192-196.

17. Ebenfelt $A$ and Lundberg $C$ : Bacterial invasion of the tonsillar tissues in acute pharyngotonsillitis and in the adenoid: $A$ preliminary study Clin Otolaryngol 1994, 19:310-313.

18. Ebenfelt $A$ and Lundberg $C$ : Cellular defence in surface secretion in acute pharyngotonsillitis Acta Otolaryngol (Stockh) 1996, I 1 6:97-103.

19. Ebenfelt A: Bacterial localization and cellular defence in pharyngotonsillitis [Thesis]: Göteborgs Universitet 1996.

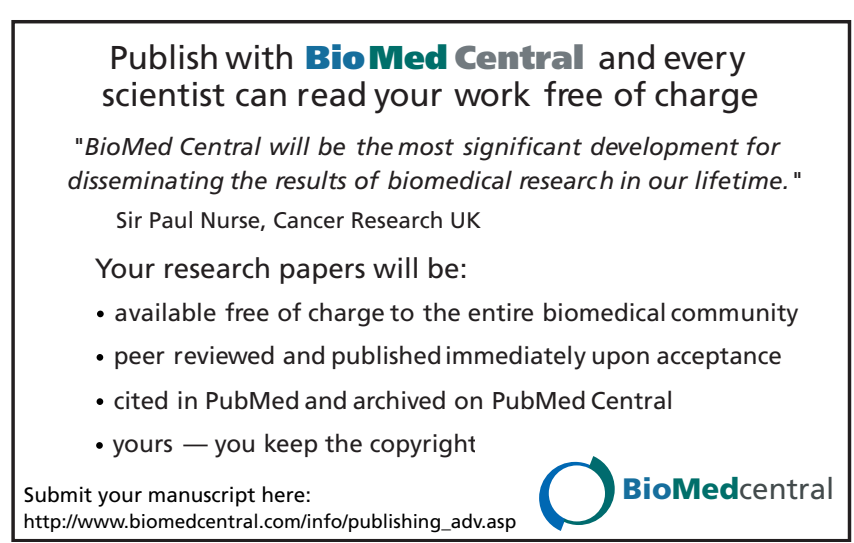

